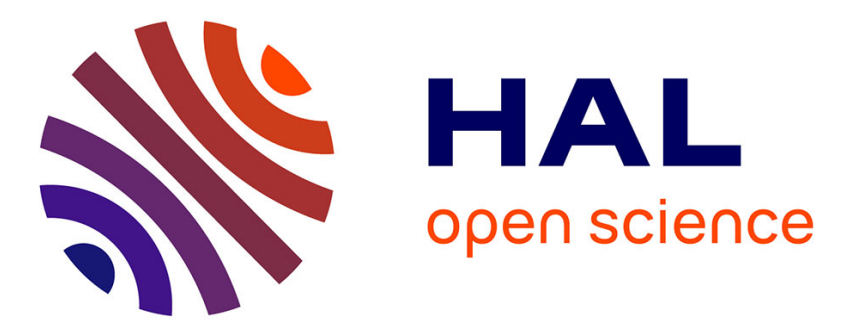

\title{
Parallel implementation of a pseudo-spectral calculation of molecular energy levels: Application to the water dimer (H O)
}

\author{
Philip Eggert, Alexandra Viel, Claude Leforestier
}

\section{- To cite this version:}

Philip Eggert, Alexandra Viel, Claude Leforestier. Parallel implementation of a pseudo-spectral calculation of molecular energy levels: Application to the water dimer (H O). Computer Physics Communications, 2000, 128 (1-2), pp.315-325. 10.1016/S0010-4655(99)00518-4 . hal-01117858

\author{
HAL Id: hal-01117858 \\ https://hal.science/hal-01117858
}

Submitted on 3 Jun 2020

HAL is a multi-disciplinary open access archive for the deposit and dissemination of scientific research documents, whether they are published or not. The documents may come from teaching and research institutions in France or abroad, or from public or private research centers.
L'archive ouverte pluridisciplinaire HAL, est destinée au dépôt et à la diffusion de documents scientifiques de niveau recherche, publiés ou non, émanant des établissements d'enseignement et de recherche français ou étrangers, des laboratoires publics ou privés. 


\title{
Parallel implementation of a pseudo-spectral calculation of molecular energy levels : Application to the water dimer $\left(\mathrm{H}_{2} \mathrm{O}\right)_{2}$
}

\author{
Philip Eggert ${ }^{a}$, Alexandra Viel ${ }^{b}$, and Claude Leforestier ${ }^{\ddagger}$ \\ LSDSMS (UMR 5636) - CC 014 \\ Université Montpellier 2, \\ 34095 Montpellier Cedex 5 (France)
}

\begin{abstract}
Parallel implementation of an iterative determination of energy levels of large molecular systems is presented. The basic step consists in acting the Hamiltonian operator $\mathbf{H}$ on a wavefunction $\mathbf{u}$, and is achieved by means of a Pseudo Spectral Split Hamiltonian scheme (C.leforestier et al.,J.Chem.Phys.,106,8527(1997)). The potential term evaluation V.u, which corresponds by far to the most time consuming part in the sequential code, has been distributed over all the processors. Application to the water dimer $\left(\mathrm{H}_{2} \mathrm{O}\right)_{2}$ Vibration-Rotation spectrum shows a very good parallel efficiency up to 64 processors.
\end{abstract}

\footnotetext{
${ }^{a}$ Present address :Institut für Physikalische und Theoretische Chemie, Freie Universität Berlin, D-14195 Berlin (Germany)

b Present address :Department of Chemistry, University of California, Berkeley, CA 94720-1460

‡ LEFORES@LSD.UNIV-MONTP2.FR
} 


\section{INTRODUCTION}

Due to continuing progress in experimental methods, molecular spectra become more detailed, but on the other hand more difficult to interpret. Among a few examples, one can cite

- the isomerization of acetylene in vinyldiene[40];

- the Stimulated Emission Pumping spectrum of the fluoryl formide molecule HFCO[41]; these spectra revealed the existence of bright states which can be assigned as $\left(0, n_{2}, 0,0,0, n_{6}, J, K_{a}, K_{c}\right)$ in the range $13,000-20,000 \mathrm{~cm}^{-1}$ of internal excitation.

- the molecular beam experiments on water clusters[42]; using tunable far IR lasers, these authors were able to characterize clusters $\left(\mathrm{H}_{2} \mathrm{O}\right)_{n}$ up to the heptamer $(\mathrm{n}=7)$.

The traditional approach to molecular spectra calculations consists in first expressing the Hamiltonian operator in some basis set, and then diagonalizing the resulting matrix. Such a method can only be used for molecular systems comprising a few thousands states at most $\left(N \leq 10^{4}\right)$, due to core memory limitations. These dimensions hardly suffice to handle highly excited triatomic molecules[43], as typically 10-30\% of the eigenvalues are converged with respect to the molecular basis set size.

In recent years, an alternate approach called the direct method has emerged as a powerful tool. By direct one means here a method which only requires acting the Hamiltonian operator $\mathbf{H}$ on some wavefunction, without the need of the associated matrix. First implementations were made by Feit and Fleck[44] using the Split Operator method, followed by Kosloff and Kosloff[45] by means of the Chebychev time propagator[46]. This approach relies on using two different representations of the wavefunction, and has been shown able to achieve very high accuracy[47]. Another route has been followed by Cederbaum and coworkers[48], and by Wyatt and coworkers[49], both of them using the Lanczos algorithm[50]. These authors used a unique normal mode description of the molecular system, which leads to an easy computation of the missing terms $\sum_{i \leq j \leq k} V_{i j k} Q_{i} Q_{j} Q_{k}+\ldots$ In all these methods, only a few vectors have to be kept in core memory, which allows one to consider ultra large basis sets.

The direct approach became very popular due to the emergence of the Discrete Variable Representation (DVR) method of Light and coworkers[51]. In this method, one emphasizes the grid representation in which the potential is diagonal. The direct DVR approach has been used in conjunction with iterative schemes such as 
- the Lanczos algorithm[50] for low lying [52] or resonant states[53] of triatomic systems;

- the Filter Diagonalization method[54] for highly excited[55] or resonant states[56, 57] of molecules;

- the GMRes linear solver[58, 59] for Cumulative Reaction Probabilities[60, 61] or photodissociation spectra[62]

to cite only a few examples. The direct DVR method can result in a broad spectrum of the Hamiltonian operator when use is made of curvilinear coordinates. The reason is due to the presence of almost singular terms in the kinetic energy operator, such as $1 / \sin ^{2} \theta \partial^{2} \partial \phi^{2}$. A DVR representation of the $\theta$ variable will badly behave close to $\theta=0$ or $\theta=\pi$, as the correct asymptotic form of the solution is not enforced[63].

The next step in the development of direct methods is due to Corey and coworkers[64]. In a very important paper, they showed how one can transform between a spherical harmonics basis set and a two dimensional grid associated to the polar angles. The key point of this transformation is that it treats exactly the apparent singularity of the kinetic energy operator $\left(1 / \sin ^{2} \theta \partial^{2} / \partial \phi^{2}\right)$ that appears in a pure grid description. As a result, the spectrum of the kinetic energy operator displays the smallest possible range. This paper established an efficient handling of a multidimensional grid associated to general non-direct product basis sets. The essential departure from Light's original formulation[51] is that one no longer seeks a unitary transform between the two representations. As a result, the two representations are no longer equivalent, the spectral representation becoming the primary one as it is more compact [65-69].

The Corey-Lemoine approach[64], adapted to spherical harmonics, has been generalized by Leforestier[66] to the case of Wigner functions. It consists of a Jacobi transform followed by a double Fourier transform. In order to maintain the variational principle within the scheme, care has been taken to remove the aliasing terms resulting from evaluating the potential on a grid. This has been realized by using a grid size significantly larger than the spectral representation dimension. This Pseudo-Spectral Split Hamiltonian (PSSH) method has been applied by one of us (C.L.) [66] to the computation of the VRT states of the Ar- $\mathrm{H}_{2} \mathrm{O}$ van der Waals complex, and was shown to be both very efficient and versatile with respect to the form of the potential, as well has having minimal storage requirements. This approach has been further pursued in order to compute the Vibration-Rotation spectrum of the water dimer[71, 72]. It consists in a generalization to two rotating rigid water molecules of the 
formulation previously developed for the $\mathrm{Ar}-\mathrm{H}_{2} \mathrm{O}$ system.

In this paper, we describe the implementation on a massively parallel computer of the PSSH code developed for the water dimer. Sec. II first introduces the basic algorithmic structure of the PSSH method. It is then shown in Sec. III how this algorithm can be efficiently implemented on a massively parallel computer. Finally, Sec. IV presents the performance of the resulting code and concludes.

\section{PSEUDO SPECTRAL SPLIT HAMILTONIAN METHOD}

The PSSH scheme is presented below through its application to the determination of the vibration-rotation energy spectrum of the water dimer. As the basic step of the algorithm consists in acting the Hamiltonian operator $\mathbf{H}$ on some wavefunction, this method can also be used in conjunction with other iterative schemes such as time propagation, filter diagonalization or linear system resolution. Furthermore, a great many molecular systems can be handled by this method.

\section{A. Hamiltonian Operator}

We used the Brocks et al rigid rotor formulation[73] which leads to the following Hamiltonian operator

$$
\begin{aligned}
\mathbf{H}= & -\frac{\hbar^{2}}{2 \mu_{A B}} \frac{\partial^{2}}{\partial R^{2}}+\mathbf{H}_{\text {rot }}^{(A)}+\mathbf{H}_{\text {rot }}^{(B)}+\mathbf{V}\left(R, \underline{\Omega}^{(A)}, \underline{\Omega}^{(B)}\right) \\
& +\frac{1}{2 \mu_{A B} R^{2}}\left\{\mathbf{J}^{2}+\mathbf{j}^{2}-2 \mathbf{j} . \mathbf{J}\right\}
\end{aligned}
$$

where

- $R$ is the distance between the centers of mass of the two monomers $A$ and $B$, and $\mu_{A B}$ their reduced mass,

- $\mathbf{H}_{\text {rot }}^{(\alpha)}$ and $\mathbf{j}_{A}$ are respectively the rotational Hamiltonian and angular momentum of monomer $\alpha$,

- $\mathbf{j}=\mathbf{j}_{A}+\mathbf{j}_{B}$ is the coupled internal rotational angular momentum,

- $\mathbf{J}=\mathbf{j}+\mathbf{L}$ the total angular momentum $(\mathbf{L}$ is the angular momentum of the monomer centers of mass), 
- and $\underline{\Omega}^{(\alpha)} \equiv\left(\varphi^{(\alpha)}, \theta^{(\alpha)}, \chi^{(\alpha)}\right)$ represents the Euler angles defining the orientation of monomer $\alpha$ in the body fixed axes.

\section{B. Spectral Representations}

The total spectral representation is first written as the direct product

$$
\mathcal{B}_{\text {ang }} \otimes\left\{\left|S_{n}\right\rangle, n=1, N_{S}\right\}
$$

where the $S$ 's correspond to functions adapted to describe the relative motion $R$.

In order to perform the calculations, several different angular bases $\mathcal{B}_{\text {ang }}$ are used, which are described below.

1. The uncoupled basis set $\mathcal{B}_{\text {unc }}$

This basis is defined as the direct product of Wigner bases $\left\{\left|j_{\alpha}, k_{\alpha}, \omega_{\alpha}\right\rangle\right\}$ on each monomer times a Wigner basis $\{|J, \Omega, M\rangle\}$ for the total angular momentum :

$$
\mathcal{B}_{u n c}=\left\{\left|j_{A}, k_{A}, \omega_{A}\right\rangle\right\} \otimes\left\{\left|j_{B}, k_{B}, \omega_{B}\right\rangle\right\} \otimes\{|J, \Omega, M\rangle\} \quad\left(\omega_{A}+\omega_{B}=\Omega\right) .
$$

It will only be used as an intermediate between the coupled representation and the grid (see Sec.II C).

2. The coupled basis set $\mathcal{B}_{c p l}$

One can contract this angular basis by defining the coupled basis set

$$
\mathcal{B}_{c p l}=\left\{\left|j_{A} j_{B} k_{A} k_{B} ; j \Omega\right\rangle|J, \Omega, M\rangle\right\} \quad\left(\left|j_{A}-j_{B}\right| \leq j \leq j_{A}+j_{B}\right)
$$

where

$$
\left|j_{A} j_{B} k_{A} k_{B} ; j \Omega\right\rangle=\sum_{\omega_{A}+\omega_{B}=\Omega}\left\langle j_{A} j_{B} \omega_{A} \omega_{B} \mid j_{A} j_{B} j \Omega\right\rangle\left|j_{A}, k_{A}, \omega_{A}\right\rangle\left|j_{B}, k_{B}, \omega_{B}\right\rangle
$$

$\left\langle j_{A} j_{B} \omega_{A} \omega_{B} \mid j_{A} j_{B} j \Omega\right\rangle$ being a Clebsch-Gordan coefficient. This representation is used to compute the $\mathbf{H}_{\text {rot }}^{(A)}, \mathbf{H}_{\text {rot }}^{(B)}$, and Centrifugal+Coriolis terms as will be shown later on. 
3. The symmetry adapted bases $\mathcal{B}_{\text {sym }}^{(\Gamma)}$

The $G_{16}$ Permutation-Inversion symmetry of the $\left(\mathrm{H}_{2} \mathrm{O}\right)_{2}$ complex[74] allows us to subdivide the angular basis set into 10 symmetry adapted bases, corresponding to the different Irreducible Representations (Irrep) $\Gamma$ 's. The way to construct symmetry adapted vectors $\left|j_{A} j_{B} k_{A} k_{B} ; j \Omega, \Gamma\right\rangle$ from the coupled basis vectors,

$$
\begin{aligned}
\left|j_{A} j_{B} k_{A} k_{B} ; j \Omega, \Gamma\right\rangle & =c_{1}^{(\Gamma)}\left|j_{A} j_{B} k_{A} k_{B} ; j \Omega\right\rangle+c_{2}^{(\Gamma)}\left|j_{B} j_{A} k_{B} k_{A} ; j \bar{\Omega}\right\rangle \\
& +c_{3}^{(\Gamma)}\left|j_{A} j_{B} \bar{k}_{A} \bar{k}_{B} ; j \bar{\Omega}\right\rangle+c_{4}^{(\Gamma)}\left|j_{B} j_{A} \bar{k}_{B} \bar{k}_{A} ; j \Omega\right\rangle
\end{aligned}
$$

where $\bar{k} \equiv-k$, is given in reference 71 . In the following, we will use $\left\{|\nu\rangle_{\Gamma}, \nu=1, N_{\Gamma}\right\}$ as a shorthand notation for the elements of the symmetry adapted basis corresponding to the Irrep $\Gamma$.

\section{Grid Representation}

The different spectral representations defined just above allows one to easily compute the effect of the various parts of the Hamiltonian operator, except for the potential. The complete scheme will be presented in the next subsection. We now discuss how the potential term is handled.

The way to evaluate the potential term consists in using a general pseudo-spectral method, as defined by Friesner[75] for the case of electronic structure calculations. This method resorts to an intermediate grid representation, tantamount to making use of a quadrature rule in order to compute the matrix elements in the spectral representation. The potential function depends on the 6 coordinates $\left\{\varphi=\varphi^{A}-\varphi^{B}, \chi^{A}, \chi^{B}, \theta^{A}, \theta^{B}, R\right\}$, where $\left(\varphi^{A}, \theta^{A}, \chi^{A}\right)$ represents the three Euler angles orientating monomer $A$ in the BF frame. The grid representation corresponds to the set of values $\left\{\Psi_{g q s \alpha \beta z}\right\}$ taken by the wave function $\Psi$ on the $6 \mathrm{D}$ grid $\left\{\varphi_{g} \times \chi_{q}^{A} \times \chi_{s}^{B} \times \theta_{\alpha}^{A} \times \theta_{\beta}^{B} \times R_{z}\right\}$, restricted to grid points where the potential energy is lower than some threshold $V_{\text {max }}$ :

$$
V\left(\varphi_{g}, \chi_{q}^{A}, \chi_{s}^{B}, \theta_{\alpha}^{A}, \theta_{\beta}^{B}, R_{z}\right) \leq V_{\max }
$$

The most convenient basis set to start from, in order to transform to the grid representation, corresponds to the uncoupled one $\mathcal{B}_{\text {ang }} \otimes\left\{\left|S_{n}\right\rangle, n=1, N_{S}\right\}$. That is, starting from a 
wave function expressed as

$$
\left|\Psi^{J M}\right\rangle=\sum_{j_{A}, k_{A}, j_{B}, k_{B}, \omega_{A}+\omega_{B}=\Omega, n} \Psi_{j_{A} k_{A} \omega_{A} j_{B} k_{B} \omega_{B} n}^{J M}\left|j_{A}, k_{A}, \omega_{A}\right\rangle\left|j_{B}, k_{B}, \omega_{B}\right\rangle|J, \Omega, M\rangle\left|S_{n}\right\rangle,
$$

one wants to compute its amplitudes $\left\{\Psi^{J M}\left(\varphi_{g}, \chi_{q}^{A}, \chi_{s}^{B}, \theta_{\alpha}^{A}, \theta_{\beta}^{B}, R_{z}\right) \equiv \Psi_{g q s \alpha \beta z}^{J M}\right\}$ on the grid. This spectral-to-grid transform is performed in several successive steps.

The first step consists in switching from the radial basis set $\left\{\left|S_{n}\right\rangle, n=1, N_{S}\right\}$ to a grid $\left\{R_{z}=R_{\min }+z \Delta R\right\}$ by means of some orthogonal collocation matrix $U_{z n}^{(R)}=\left\langle R_{z} \mid S_{n}\right\rangle$. This matrix allows one to define the intermediate representation $\left\{\Psi_{j_{A} k_{A} \omega_{A} j_{B} k_{B} \omega_{B} z}^{J M}\right.$ by means of the transformation

$$
\Psi_{j_{A} k_{A} \omega_{A} j_{B} k_{B} \omega_{B} z}^{J M}=\sum_{n} U_{z n}^{(R)} \Psi_{j_{A} k_{A} \omega_{A} j_{B} k_{B} \omega_{B} z}^{J M}
$$

the inverse (grid to spectral) transform being performed by using the transpose collocation matrix.

The second step deals with the angular to Euler grid transformation. One can show[71] that the transformation from the angular spectral representation to the five dimensional grid can be realized by the following successive operations, performed at every values $\Omega=\omega_{B}+\omega_{A}$ $(-J \leq \Omega \leq J):$

i) transform to the $\left\{\theta_{\alpha}^{A} \times \theta_{\beta}^{B}\right\}$ grid

$$
\Psi_{k_{A} \omega_{A} k_{B} \omega_{B} \alpha \beta z}^{J M \Omega}=\sum_{j_{A}, j_{B}} R_{\alpha j_{A}}^{\left(\omega_{A}, k_{A}\right)} R_{\beta j_{B}}^{\left(\omega_{B}, k_{B}\right)} \Psi_{j_{A} k_{A} \omega_{A} j_{B} k_{B} \omega_{B} z}^{J M}
$$

by means of the orthogonal collocation matrices

$$
R_{\alpha j}^{(\omega, k)}=\sqrt{\frac{2 j+1}{2}} d_{\omega k}^{j}\left(\cos \theta_{\alpha}\right) \sqrt{w_{\alpha}}
$$

$\cos \theta_{\alpha}$ and $w_{\alpha}$ being respectively the abscissae and weights of a $N_{\theta}$ points Gauss-Legendre quadrature;

ii) transform to the $\left\{\phi_{g} \times \chi_{q}^{A} \times \chi_{s}^{B}\right\}$ grid by a 3D inverse Fourier transform

$$
\left\{\Psi_{g q s \alpha \beta z}^{J M \Omega}\right\}=\mathcal{F}_{\varphi \chi^{A} \chi^{B}}^{-1}\left\{\Psi_{k_{A} \omega_{A} k_{B} \omega_{B} \alpha \beta z}^{J M \Omega}\right\}
$$

Going back to the $\left\{\left|j_{A}, k_{A}, \omega_{A}\right\rangle\left|j_{B}, k_{B}, \omega_{B}\right\rangle\right\}$ representation is realized by applying the inverse transforms in reverse order, i.e. i i) then i). 


\section{The Split Hamiltonian Formulation}

It consists in repetitively applying $\mathbf{H}$ on the wave function expressed in the $\mathcal{B}_{\text {sym }}^{(\Gamma)} \otimes\left\{\left|S_{n}\right\rangle\right\}$ basis set,

$$
\left|\Psi^{J M \Gamma}\right\rangle=\sum_{\nu \Omega n} \Psi_{\nu \Omega n}^{J M \Gamma}|\nu, \Omega\rangle|J \Omega M\rangle\left|S_{n}\right\rangle,
$$

in order to propagate the Lanczos scheme. The choice of the symmetry adapted basis results from the fact that it constitutes the most compact basis for expressing the Lanczos vectors $\left\{\left|u_{n}\right\rangle\right\}$. We now briefly recall the Lanczos algorithm, and then discuss the different terms into which the Hamiltonian is split, as well as their associated representations.

Starting from some initial vector $\left|u_{0}\right\rangle$, the Lanczos algorithm[50] recursively generates the Krylov space $\left\{\left|u_{n}\right\rangle n=0, N_{L}\right\}$

$$
\beta_{n+1}\left|u_{n+1}\right\rangle=\left(\mathbf{H}-\alpha_{n}\right)\left|u_{n}\right\rangle-\beta_{n}\left|u_{n-1}\right\rangle
$$

where $\alpha_{n}=\left\langle u_{n}|\mathbf{H}| u_{n}\right\rangle$ and $\beta_{n+1}=\left\langle u_{n+1}|\mathbf{H}| u_{n}\right\rangle$ are respectively the diagonal and off-diagonal terms of $\mathbf{H}$ in this new basis set.

In order to perform the recursions, $\mathbf{H}$ as given by Eq.1, has been split into 4 terms, namely

$$
\mathbf{H}=\mathbf{T}_{R}+\mathbf{H}_{A B}+\mathbf{H}_{c c}+\mathbf{V}
$$

where each term has been handled as shown below.

\section{Radial Kinetic Energy $\mathbf{T}_{R}$}

This term, $\mathbf{T}_{R}=-\hbar^{2} / 2 \mu_{A B} \cdot \partial^{2} / \partial R^{2}$, is associated to the $\left[T_{n n^{\prime}}\right]$ matrix in the initial representation (Eq.13), resulting in the effect

$$
\mathbf{T}_{R}\left|\Psi^{J M \Gamma}\right\rangle=\sum_{\nu \Omega n n^{\prime}} T_{n n^{\prime}} \Psi_{\nu \Omega n^{\prime}}^{J M \Gamma}|\nu, \Omega\rangle|J \Omega M\rangle\left|S_{n}\right\rangle
$$

2. Monomer rotational terms $\mathbf{H}_{A B}=\mathbf{H}_{A}+\mathbf{H}_{B}$

The rotational kinetic energy term $\mathbf{H}_{\alpha}$ displays analytic expressions in a Wigner basis set $\{|j k \omega\rangle\}$ given by

$$
\mathbf{H}_{\alpha}|j k \omega\rangle=\left\{\frac{1}{2}\left(B_{x}+B_{y}\right)\left(j(j+1)-k^{2}\right)+B_{z} k^{2}\right\}|j k \omega\rangle
$$




$$
\begin{aligned}
& +\frac{1}{4}\left(B_{x}-B_{y}\right) F_{j k}^{+}|j k+2 \omega\rangle \\
& +\frac{1}{4}\left(B_{x}-B_{y}\right) F_{j k}^{-}|j k-2 \omega\rangle
\end{aligned}
$$

where $F_{j k}^{ \pm}=[j(j \pm 1)-k(k \pm 1)]^{\frac{1}{2}}[j(j \pm 1)-(k \pm 1)(k \pm 2)]^{\frac{1}{2}}$. In order to exploit the above relations, one has to switch to the coupled basis set $\mathcal{B}_{c p l}$

$$
\left|\Psi^{J M \Gamma}\right\rangle=\sum_{j_{A} k_{A} j_{B} k_{B} j \Omega n} \Psi_{j_{A} k_{A} j_{B} k_{B} j \Omega n}^{J M \Gamma}\left|j_{A} j_{B} k_{A} k_{B} ; j \Omega\right\rangle|J, \Omega, M\rangle\left|S_{n}\right\rangle .
$$

As Eq.17 only involves changes in the $k$ index, the $\mathbf{H}_{\alpha}$ terms can be directly applied in this representation.

\section{Coriolis and centrifugal term $\mathbf{H}_{C C}$}

This term, $\mathbf{H}_{C C}=1 / 2 \mu_{A B} R^{2}\left\{\mathbf{J}^{2}+\mathbf{j}^{2}-2 \mathbf{j} . \mathbf{J}\right\}$ also displays simple analytic expressions in the coupled angular basis set

$$
\begin{aligned}
\mathbf{H}_{C C}|j \Omega\rangle|J, \Omega, M\rangle= & {\left[J(J+1)+j(j+1)-2 \Omega^{2}\right]|j \Omega\rangle|J, \Omega, M\rangle } \\
& +C_{J \Omega}^{+} C_{j \Omega}^{+}|j \Omega+1\rangle|J, \Omega+1, M\rangle \\
& +C_{J \Omega}^{-} C_{j \Omega}^{-}|j \Omega-1\rangle|J, \Omega-1, M\rangle
\end{aligned}
$$

where $C_{j \Omega}^{ \pm}=[j(j \pm 1)-\Omega(\Omega \pm 1)]^{\frac{1}{2}}$. The $1 / 2 \mu_{A B} R^{2}$ factor in front of $\mathbf{H}_{C C}$ is handled by switching to the $\left\{R_{z}\right\}$ grid, where it is diagonal, by means of Eq.9

$$
\left\{\Psi_{j_{A} k_{A} j_{B} k_{B} j \Omega n}^{J M \Gamma}\right\} \rightarrow\left\{\Psi_{j_{A} k_{A} j_{B} k_{B} j \Omega z}^{J M \Gamma}\right\}
$$

\section{Potential term $\mathbf{V}$}

As discussed before, this last term is diagonal in the $6 \mathrm{D}$ grid times the total angular momentum representation $\left\{\varphi_{g} \times \chi_{q}^{A} \times \chi_{s}^{B} \times \theta_{\alpha}^{A} \times \theta_{\beta}^{B} \times R_{z}\right\} \otimes\{|J, \Omega, M\rangle\}$, which can be reached, as discussed in Sec.II C, by means of Eq.(10-12)

$$
\left\{\Psi_{j_{A} k_{A} j_{B} k_{B} j \Omega p}^{J M \Gamma}\right\} \rightarrow\left\{\Psi_{\alpha q \beta s g p}^{J M \Gamma \Omega} \cdot\right\}
$$

Once a term of $\mathbf{H}$ has been applied in its own representation, the result is transformed back to the symmetrized times the radial functions basis set $\mathcal{B}_{\text {sym }} \otimes\left\{\left|S_{n}\right\rangle\right\}$ in which the Lanczos vectors are expressed. 


\section{PARALLEL IMPLEMENTATION OF THE PSSH SCHEME}

Two distinct steps can be distinguished in the PSSH scheme :

(i) the first one concerns the determination of the effective six-dimensional grid to be used according to the energy criterion (Eq. 7);

(ii) the second step consists in acting the Hamiltonian operator $\mathbf{H}$ on a given wavefunction $\mathbf{u}_{n}$ (Eq. 14).

In fact, a PSSH calculation can be viewed as step (i) executed only once, followed by step (ii) executed several times until convergence of the energy levels, as shown in Fig. 1.

Figure 1 near here

Before describing the parallel implementation of steps (i) and (ii), we first discuss the general organization of the code. The Single Program Multiple Data (SPMD) model has been used as it supports both the Parallel Virtual Machine (PVM) and Message Passing Interface (MPI) protocols. Within this model, processor \#0 is mainly used as a driver for the whole program, and can also effectuate some very fast calculations which will be indicated later on. Conversely, processors \#1 to \#N-1 are dedicated to parallel executions of steps (i) and (ii) as described now.

\section{A. Effective Grid Definition}

In order to define the effective grid satisfying Eq. 7, a systematic sampling of the whole six dimensional space has to be performed. Due to the two-step structure, Eq .9-10 followed by Eq. 12, of the spectral to grid transformation the $6 \mathrm{D}$ grid $\left\{\varphi_{g} \times \chi_{q}^{A} \times \chi_{s}^{B} \times \theta_{\alpha}^{A} \times \theta_{\beta}^{B} \times R_{z}\right\}$ is partitioned into three dimensional subgrids $\left\{\varphi_{g} \times \chi_{q}^{A} \times \chi_{s}^{B}\right\}_{\alpha \beta z}$, each one corresponding to a given triplet $\left(\theta_{\alpha}^{A}, \theta_{\beta}^{B}, R_{z}\right) \equiv(\alpha \beta z)$ while the $\varphi, \chi^{A}$ and $\chi^{B}$ angles vary over their definition ranges. The total number $N_{\theta}^{2} \times N_{R}$ of subgrids (or triplets) to be searched is then evenly distributed over the $N-1$ processors. This number is typically of the order of $10^{3}$, far greater than $N-1$, which leads to an excellent load balancing between the different processors.

This primary partitionning of the six dimensional grid cannot be retained for step (ii), as some effective subgrids $(\alpha \beta z)$ are essentially empty : all their grid points correspond to a 
potential energy greater than the threshold $V_{\max }$. It can be shown, from the structure of the pseudo spectral algorithm, that the computational cost associated to acting $\mathbf{H}$ on a vector $\mathbf{u}$ depends primarily on the number of non empty subgrids, and to a lesser extent on their density $\rho_{\alpha \beta z}$. An empty subgrid is associated to a zero density, while a full one corresponds to a density value of 1 . Consequently, to each subgrid we assign an effective $\operatorname{cost} \sigma_{\alpha \beta z}$ given by

$$
\sigma_{\alpha \beta z}=\rho_{\alpha \beta z}^{\eta}
$$

The dependence of this function on the $\eta$ parameter is shown in Fig.2

Figure 2 near here

A second partitioning of the six dimensional grid is then performed, taking into account this effective cost for each subgrid. That is, the global cost $\sum_{\alpha \beta z} \sigma_{\alpha \beta z}$ is evenly split over processors \#1 to \#N-1, with the additional constraint that no subgrid extends over two processors. This latter requirement is only used for simplifying the implementation of the scheme. It was found that a low value of the $\eta$ parameter, $\eta \sim 0.2$, leads to a better load balancing of step (ii).

\section{B. Acting the Hamiltonian Operator}

The scheme, as presented in Sec.II D, consists in acting succesively the four terms of the operator (Eq. 15). In order to decide if all terms have to be implemented in a parallel way, Table I displays their relative CPU times in a sequential calculation. These times correspond to a basis set size typically used in the calculations $(0 \leq j \leq 10)$. It can be seen that for $J=0$ calculations only $1 \%$ of the CPU time is needed for acting the first three terms $\mathbf{T}_{R}+\mathbf{H}_{A B}+\mathbf{H}_{c c}$, while $99 \%$ is actually spent acting the potential term $\mathbf{V}$. The reason stems from the fact that acting $\mathbf{V}$ requires to reexpress the wavefunction on the six dimensional grid as described in Sec. II C, while $\mathbf{T}_{R}$ can be directly obtained in the initial representation $\mathcal{B}_{\text {sym }}^{(\Gamma)}$ and $\mathbf{H}_{A B}+\mathbf{H}_{c c}$ necessitate to switch to the closely related coupled angular basis set $\mathcal{B}_{\text {cpl }}$.

Table I near here 
The simplest way to implement a parallel version of the PSSH scheme consists in keeping the evaluation of the $\mathbf{T}_{R}+\mathbf{H}_{A B}+\mathbf{H}_{c c}$ terms on processor $\# 0$, while devoting processors \#1 to \#N-1 to the potential term $\mathbf{V}$ evaluation. The resulting structure, as illustrated in Fig. 3, is the following :

Figure 3 near here

(a) the $\mathbf{u}_{n}$ vector, expressed in the compact, symmetry adapted basis set (Eq. 13), is broadcasted to processors \#1 to \#N-1;

(b) each processor $p(1 \leq p \leq N-1)$ transforms the vector $\mathbf{u}_{n}$ to the grid representation $\mathbf{u}_{n}^{(p)}$ restricted to the actual subgrids $(\alpha \beta z)$ pertaining to this processor, as previously assigned in step i); after applying the local potential $\mathbf{V}^{(p)}$, the resulting vector $\mathbf{V}^{(p)} \mathbf{u}_{n}^{(p)}$ is transformed back to the symmetry adapted representation : $\rightarrow \mathbf{v}_{n}^{(p)}$; simultaneously, processor $\# 0$ starts the evaluation of terms $\mathbf{T}_{R}+\mathbf{H}_{A B}+\mathbf{H}_{c c}$ :

$$
\mathbf{u}_{n+1}:=\left(\mathbf{T}_{R}+\mathbf{H}_{A B}+\mathbf{H}_{c c}\right) \mathbf{u}_{n}-\alpha_{n} \mathbf{u}_{n}
$$

when this calculation is completed, processor \#0 switches to a waiting mode for data coming from any processor;

(c) as soon as completed, the $\mathbf{v}_{n}^{(p)}$ vectors are sent to processor \#0 which collects them in the vector $\mathbf{u}_{n+1}$ :

$$
\mathbf{u}_{n+1}:=\mathbf{u}_{n+1}+\mathbf{v}_{n}^{(p)} \quad(1 \leq p \leq N-1) .
$$

\section{PERFORMANCES AND DISCUSSION}

Fig. 4 displays the evolution of the elapsed CPU time as a function of the total number $N$ of processors used. These calculations have been done on a Cray/T3E machine running under the MPI protocol. Similar results, not shown here, have been obtained using PVM. The number $N$ of processors considered has been systematically doubled from $N=4$ to $N=128$. 
Figure 4 near here

This figure displays the performances of the whole PSSH scheme, as well as that of the grid definition step. On can first note that up to $N=32$, the elapsed times decrease faster than linearly with the number $N$ of processors. The reason stems from the fact that only $N-1$ processors are actually used in the parallel calculations, processor \#0 being assigned the role of a driver. When going from $N=4$ to $N=16$ for example, there is a fivefold increase in the number of processors in charge of the parallelized step. At larger values of $N$, one can note that the grid definition step displays a better parallel efficiency. This behavior results from the negligible amount of data transferred during this step. Conversely, the whole set of of vectors $\left\{\mathbf{v}_{n}^{(p)}, p=1 \ldots N-1\right\}$ has to be sent to processor $\# 0$ once the potential term $\mathbf{V}$ has been acted on the different processors. Using the built in procedure REDUCE did not show any significant improvement. This figure also shows that the grid evaluation represents a minor part of the whole calculation, the reason being the simplicity of the MCY potential[76] used in these tests. More intricate functions, such as the ASP potentials of Stone and coworkers[77], would lead to a two order of magnitude increase in CPU time for the grid evaluation step. One can consider that the global scheme displays an excellent parallel efficiency up to 64 processors about.

As has been already mentioned, the PSSH scheme presented in this paper displays the basic ingredients for many iterative methods used in Chemical Reaction Dynamics. Although applied in this work to bound states calculations, the Lanczos algorithm can be applied to photodissociation spectra[78]. Furthermore, in Fig. 1 the Lanzos scheme can be replaced by a Chebychev propagator[46] control section in order to perform time dependent wavepacket[47] or Filter Diagonalization[54, 55] calculations. Similarly, a GMRes[59] module would allow for calculations of Cumulative Reaction Probabilities[60, 61] or of photodissociation spectra[62].

The versatility of the parallel scheme developed in this work should find many applications in the study of molecular processes.

\section{ACKNOWLEDGMENTS}

This work was partially supported by France-Berkeley Fund, and by grants of computer time from the Institut du Développement et des Ressources en Informatique Scientifique 
(IDRIS) and from the Centre Informatique National de l'Enseignement Suprieur (CINES).

[1] Y.Chen, D.M.Jonas, C.E.Hamilton, P.G.Green, J.L.Kinsey and R.W.Field, Ber.Bunsenges.Phys.Chem. 92, 329 (1988).

[2] Y.S.Choi and C.B.Moore, J.Chem.Phys. 94, 5414 (1991).

[3] K.Liu, J.D.Cruzan, and R.J.Saykally, Science 271, 929 (1996).

[4] J.Tennyson, ComP.Phys.Comm.,current issue .

[5] M.D.Feit, J.D.Fleck, and A.Steiger, J.Comp.Phys. 47, 412 (1982).

[6] D.Kosloff and R.Kosloff, J.Comp.Phys. 52, 35 (1983).

[7] H.Tal-Ezer and R.Kosloff, J.Chem.Phys. 81, 3967 (1984).

[8] R.Kosloff, J.Phys.Chem. 92, 2087 (1988).

[9] H.Köppel, W.Domcke, and L.S.Cederbaum, Adv.Chem.Phys. 57, 57 (1984).

[10] R.A.Friesner, J.P.Brunet, R.E.Wyatt, and C.Leforestier, Int.J.Supercomputer Applications 1, 9 (1987).

[11] C.Lanczos, J.Res.Natl.Bur.Stand. 45, 255 (1950).

[12] J.C.Light, I.P.Hamilton, and J.V.Lill, J.Chem.Phys. 82, 1400 (1985).

[13] F.LeQuéré and C.Leforestier, J.Chem.Phys. 94, 1118 (1991).

[14] C.Leforestier, K.Yamashita, and N.Moiseyev, J.Chem.Phys. 103, 8468 (1995).

[15] D.Neuhauser, J.Chem.Phys. 93, 2611 (1990).

[16] M.R.Wall and D.Neuhauser, J.Chem.Phys. 102, 8011 (1995).

[17] T.P.Grozdanov, V.A.Mandelshtam, and H.S.Taylor, J.Chem.Phys. 103, 23 (1995).

[18] V.A.Mandelshtam, T.P.Grozdanov, and H.S.Taylor, J.Chem.Phys. 103, 10074 (1995).

[19] Y.Saad and M.H.Schultz, SIAM J.Sci.Stat.Comput. 7, 856 (1986).

[20] M.S.Reeves, D.C.Chatfield, and D.G.Truhlar, J.Chem.Phys. 99, 2739 (1993).

[21] T.Seideman and W.H.Miller, J.Chem.Phys. 96, 4412 (1992).

[22] T. Germann and W. Miller, J.Phys.Chem.A 101, 6358 (1997).

[23] T.Seideman, J.Chem.Phys. 98, 1989 (1993).

[24] R.M.Whitnell and J.C.Light, J.Chem.Phys. 89, 3674 (1988).

[25] G.Corey and D.Lemoine, J.Chem.Phys. 97, 4115 (1992).

[26] M.J.Bramley, J.W.Tromp, T.Carrington, and G.C.Corey, J.Chem.Phys. 100, 6175 (1994). 
[27] C.Leforestier, J.Chem.Phys. 101, 7357 (1994).

[28] D.Lemoine, J.Chem.Phys. 101, 10526 (1994).

[29] O.A.Sharafeddin and J.C.Light, J.Chem.Phys. 102, 3622 (1995).

[30] G.Corey and J.W.Tromp, J.Chem.Phys. 103, 1812 (1995).

[31] J.K.Cullum and R.A.Willoughby, Lanczos Algorithms for Large Symmetric Eigenvalues Computations (Birkhäuser, Boston, 1985).

[32] C.Leforestier, L.B.Braly, K.Liu, M.J.Elrod and R.J.Saykally,J.Chem.Phys. 106, 8527 (1997).

[33] R.S.Fellers, L.B.Braly, M.G.Brown, C.Leforestier and R.J.Saykally, J.Chem.Phys. 110, 6306 (1999).

[34] G.Brocks, A. der Avoird, B.T.Sutcliffe, and J.Tennyson, Mol.Phys. 50, 1025 (1983).

[35] T.R.Dyke, J.Chem.Phys. 66, 482 (1977).

[36] R.A.Friesner, J.Chem.Phys. 85, 1462 (1986).

[37] O.Matsuoka, E.Clementi, and M.Yoshimine, J.Chem.Phys. 64, 1351 (1976).

[38] C.Millot and A.J.Stone, Mol.Phys. 77, 439 (1992).

[39] M.Alacid and C.Leforestier, Int.J.Quantum Chem. 68, 317 (1998).

[40] Y.Chen, D.M.Jonas, C.E.Hamilton, P.G.Green, J.L.Kinsey and R.W.Field, Ber.Bunsenges.Phys.Chem. 92, 329 (1988).

[41] Y.S.Choi and C.B.Moore, J.Chem.Phys. 94, 5414 (1991).

[42] K.Liu, J.D.Cruzan, and R.J.Saykally, Science 271, 929 (1996).

[43] J.Tennyson, ComP.Phys.Comm.,current issue .

[44] M.D.Feit, J.D.Fleck, and A.Steiger, J.Comp.Phys. 47, 412 (1982).

[45] D.Kosloff and R.Kosloff, J.Comp.Phys. 52, 35 (1983).

[46] H.Tal-Ezer and R.Kosloff, J.Chem.Phys. 81, 3967 (1984).

[47] R.Kosloff, J.Phys.Chem. 92, 2087 (1988).

[48] H.Köppel, W.Domcke, and L.S.Cederbaum, Adv.Chem.Phys. 57, 57 (1984).

[49] R.A.Friesner, J.P.Brunet, R.E.Wyatt, and C.Leforestier, Int.J.Supercomputer Applications 1, 9 (1987).

[50] C.Lanczos, J.Res.Natl.Bur.Stand. 45, 255 (1950).

[51] J.C.Light, I.P.Hamilton, and J.V.Lill, J.Chem.Phys. 82, 1400 (1985).

[52] F.LeQuéré and C.Leforestier, J.Chem.Phys. 94, 1118 (1991).

[53] C.Leforestier, K.Yamashita, and N.Moiseyev, J.Chem.Phys. 103, 8468 (1995). 
FIG. 1. Simplified flow chart of the PSSH code : (i) determination of the effective grid $\left\{\varphi_{g} \times \chi_{q}^{A} \times\right.$ $\left.\chi_{s}^{B} \times \theta_{\alpha}^{A} \times \theta_{\beta}^{B} \times R_{z}\right\}$ to be used later on; (ii) propagation of the initial vector $\mathbf{u}_{0}$.

[54] D.Neuhauser, J.Chem.Phys. 93, 2611 (1990).

[55] M.R.Wall and D.Neuhauser, J.Chem.Phys. 102, 8011 (1995).

[56] T.P.Grozdanov, V.A.Mandelshtam, and H.S.Taylor, J.Chem.Phys. 103, 23 (1995).

[57] V.A.Mandelshtam, T.P.Grozdanov, and H.S.Taylor, J.Chem.Phys. 103, 10074 (1995).

[58] Y.Saad and M.H.Schultz, SIAM J.Sci.Stat.Comput. 7, 856 (1986).

[59] M.S.Reeves, D.C.Chatfield, and D.G.Truhlar, J.Chem.Phys. 99, 2739 (1993).

[60] T.Seideman and W.H.Miller, J.Chem.Phys. 96, 4412 (1992).

[61] T. Germann and W. Miller, J.Phys.Chem.A 101, 6358 (1997).

[62] T.Seideman, J.Chem.Phys. 98, 1989 (1993).

[63] R.M.Whitnell and J.C.Light, J.Chem.Phys. 89, 3674 (1988).

[64] G.Corey and D.Lemoine, J.Chem.Phys. 97, 4115 (1992).

[65] M.J.Bramley, J.W.Tromp, T.Carrington, and G.C.Corey, J.Chem.Phys. 100, 6175 (1994).

[66] C.Leforestier, J.Chem.Phys. 101, 7357 (1994).

[67] D.Lemoine, J.Chem.Phys. 101, 10526 (1994).

[68] O.A.Sharafeddin and J.C.Light, J.Chem.Phys. 102, 3622 (1995).

[69] G.Corey and J.W.Tromp, J.Chem.Phys. 103, 1812 (1995).

[70] J.K.Cullum and R.A.Willoughby, Lanczos Algorithms for Large Symmetric Eigenvalues Computations (Birkhäuser, Boston, 1985).

[71] C.Leforestier, L.B.Braly, K.Liu, M.J.Elrod and R.J.Saykally,J.Chem.Phys. 106, 8527 (1997).

[72] R.S.Fellers, L.B.Braly, M.G.Brown, C.Leforestier and R.J.Saykally, J.Chem.Phys. 110, 6306 (1999).

[73] G.Brocks, A. der Avoird, B.T.Sutcliffe, and J.Tennyson, Mol.Phys. 50, 1025 (1983).

[74] T.R.Dyke, J.Chem.Phys. 66, 482 (1977).

[75] R.A.Friesner, J.Chem.Phys. 85, 1462 (1986).

[76] O.Matsuoka, E.Clementi, and M.Yoshimine, J.Chem.Phys. 64, 1351 (1976).

[77] C.Millot and A.J.Stone, Mol.Phys. 77, 439 (1992).

[78] M.Alacid and C.Leforestier, Int.J.Quantum Chem. 68, 317 (1998). 
TABLE I. CPU times given in seconds for an IBM Risc 6000/397 computer.

\begin{tabular}{ccc} 
Term & $\mathrm{J}=0$ & $\mathrm{~J}=1$ \\
\hline $\mathbf{T}_{R}$ & $\leq 0.01$ & 0.02 \\
$\mathbf{H}_{A B}+\mathbf{H}_{c c}$ & 0.21 & 1.40 \\
$\mathbf{V}$ & 18.9 & 61.0
\end{tabular}

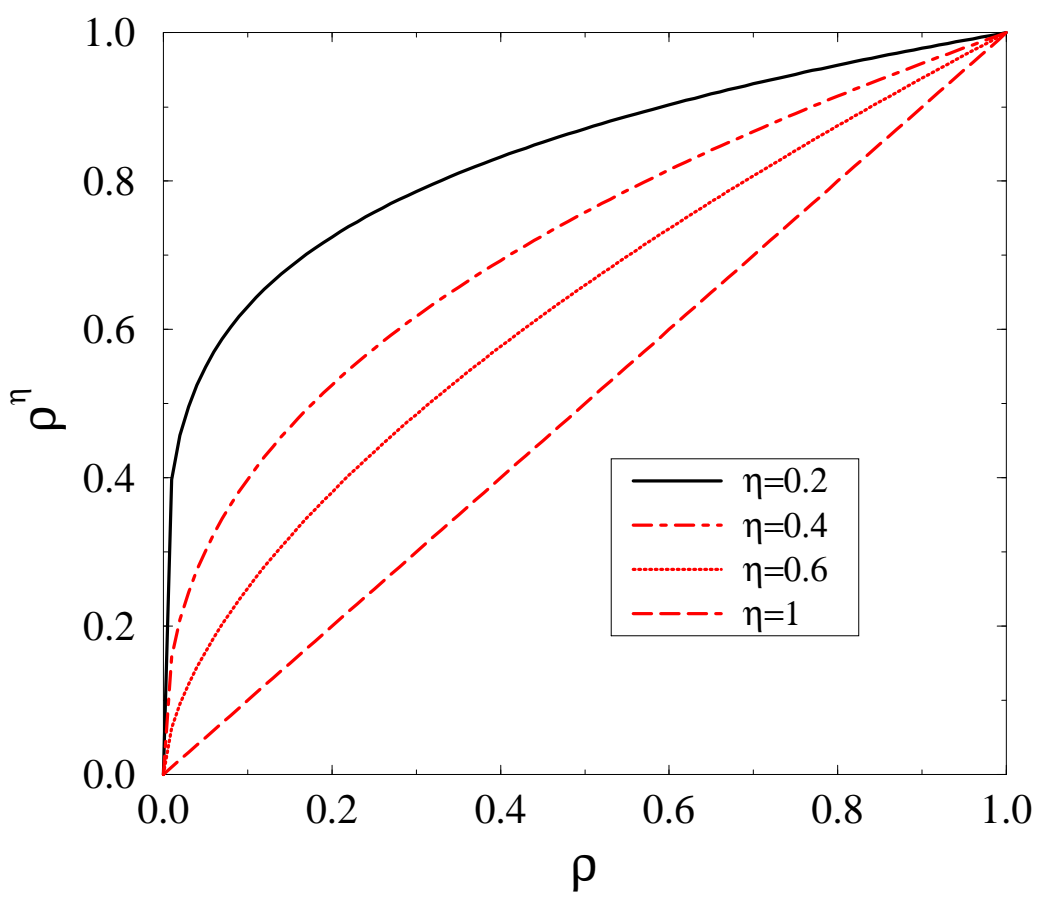

FIG. 2. Effective cost $\sigma_{\alpha \beta z}=\rho_{\alpha \beta z}^{\eta}$ associated to a subgrid $(\alpha \beta z)$ as a function of the $\eta$ parameter. $\rho$ represents the density of points kept in the calculation. 
FIG. 3. Parallel implementation of the propagation step (ii) :

(a) processor \#0 : broadcast of the $\mathbf{u}_{n}$ vector; processors \#1 to \#N-1 : waiting for data.

(b) processor \#0 : calculation of the local contribution $\left(\mathbf{T}_{R}+\mathbf{H}_{A B}+\mathbf{H}_{c c}\right) \mathbf{u}_{n}-\alpha_{n} \mathbf{u}_{n}$ to vector $\mathbf{u}_{n+1}$; processors $\# 1$ to $\# \mathrm{~N}-1$ : after transforming the $\mathbf{u}_{n}$ vector to the locally defined grid, $\rightarrow$ $\mathbf{u}_{n}^{(p)}$, and applying the potential term, the resulting vector $\mathbf{V}^{(p)} \cdot \mathbf{u}_{n}^{(p)}$ is transformed to the initial representation $\rightarrow \mathbf{v}_{n}^{(p)}$.

(c) processors \#1 to \#N-1 : sending the partial results $\mathbf{v}_{n}^{(p)}$ to processor \#0 for summing up in $\mathbf{u}_{n+1}$.

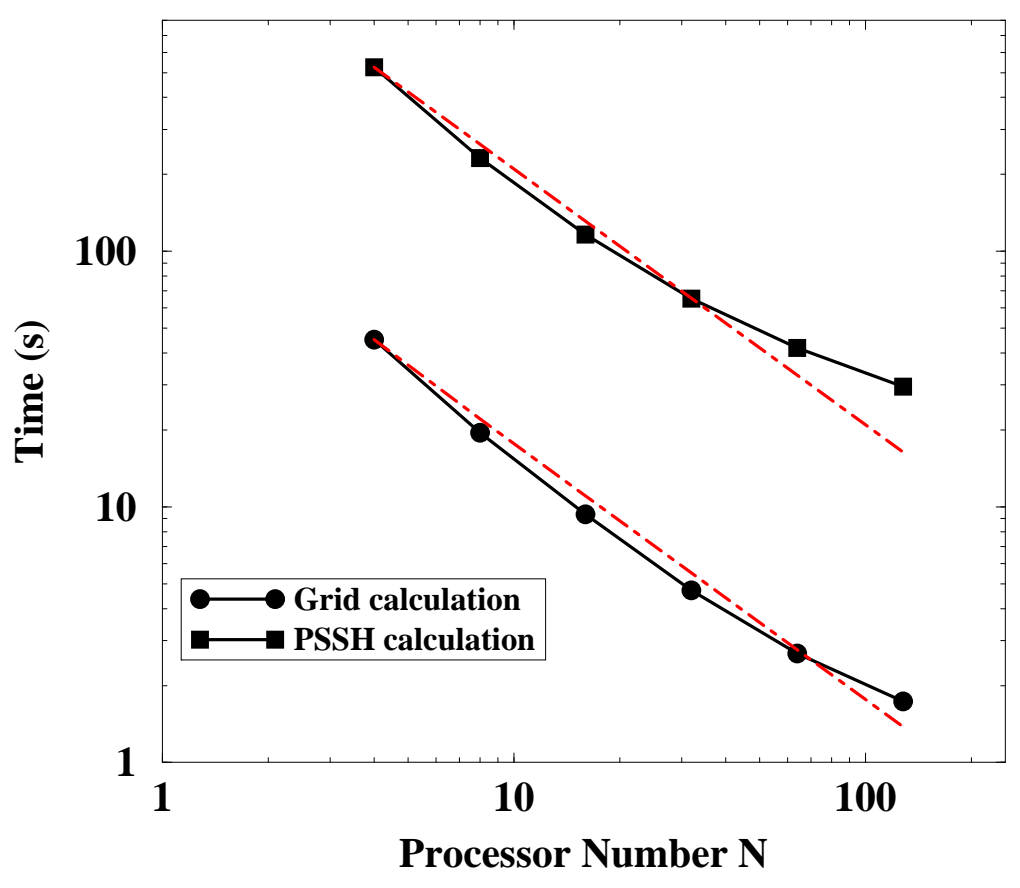

FIG. 4. Evolution of the elapsed CPU time as a function of the number $N$ of processors for both the global scheme (PSSH) and the grid definition (Grid). 


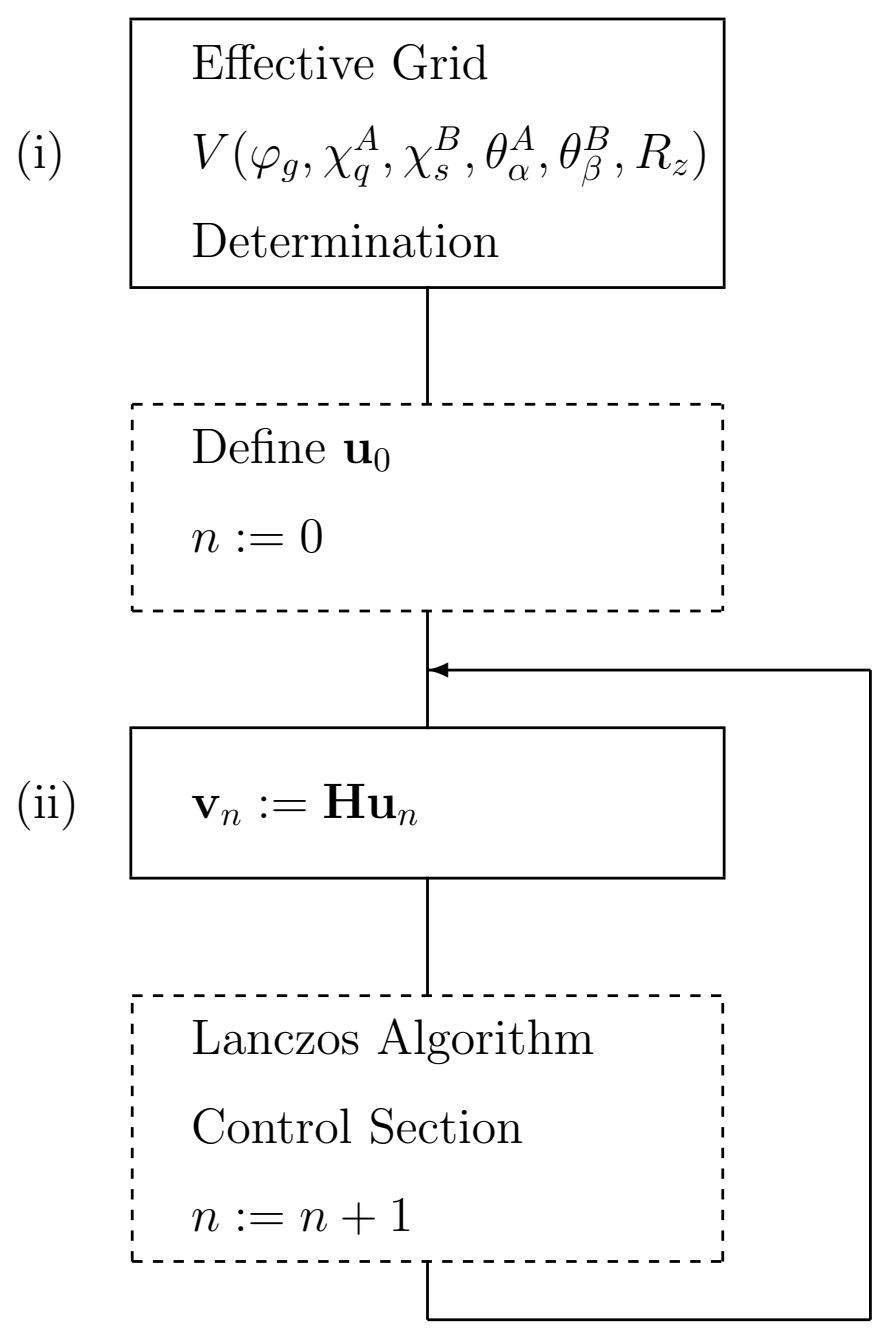


(a)

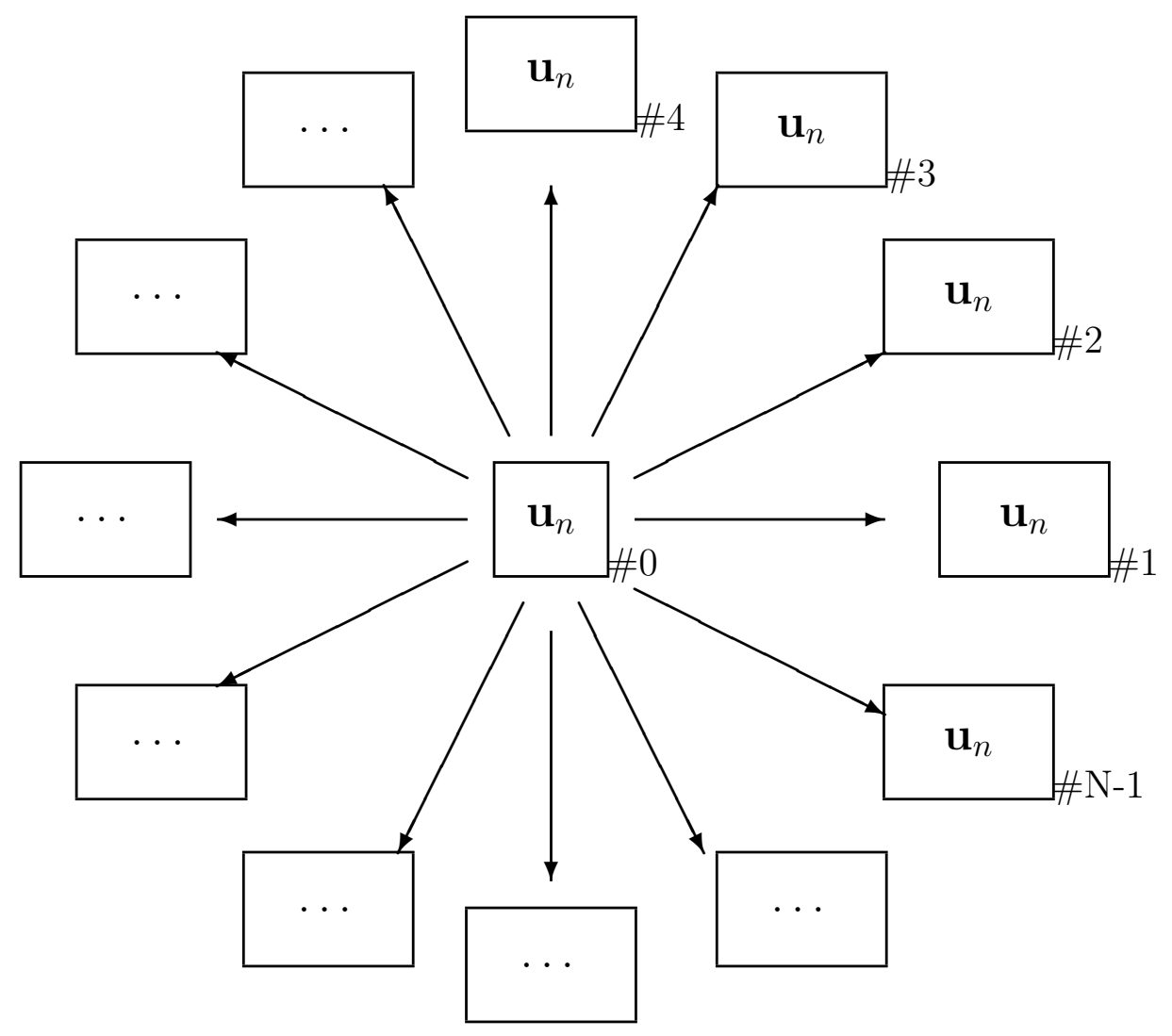


(b)

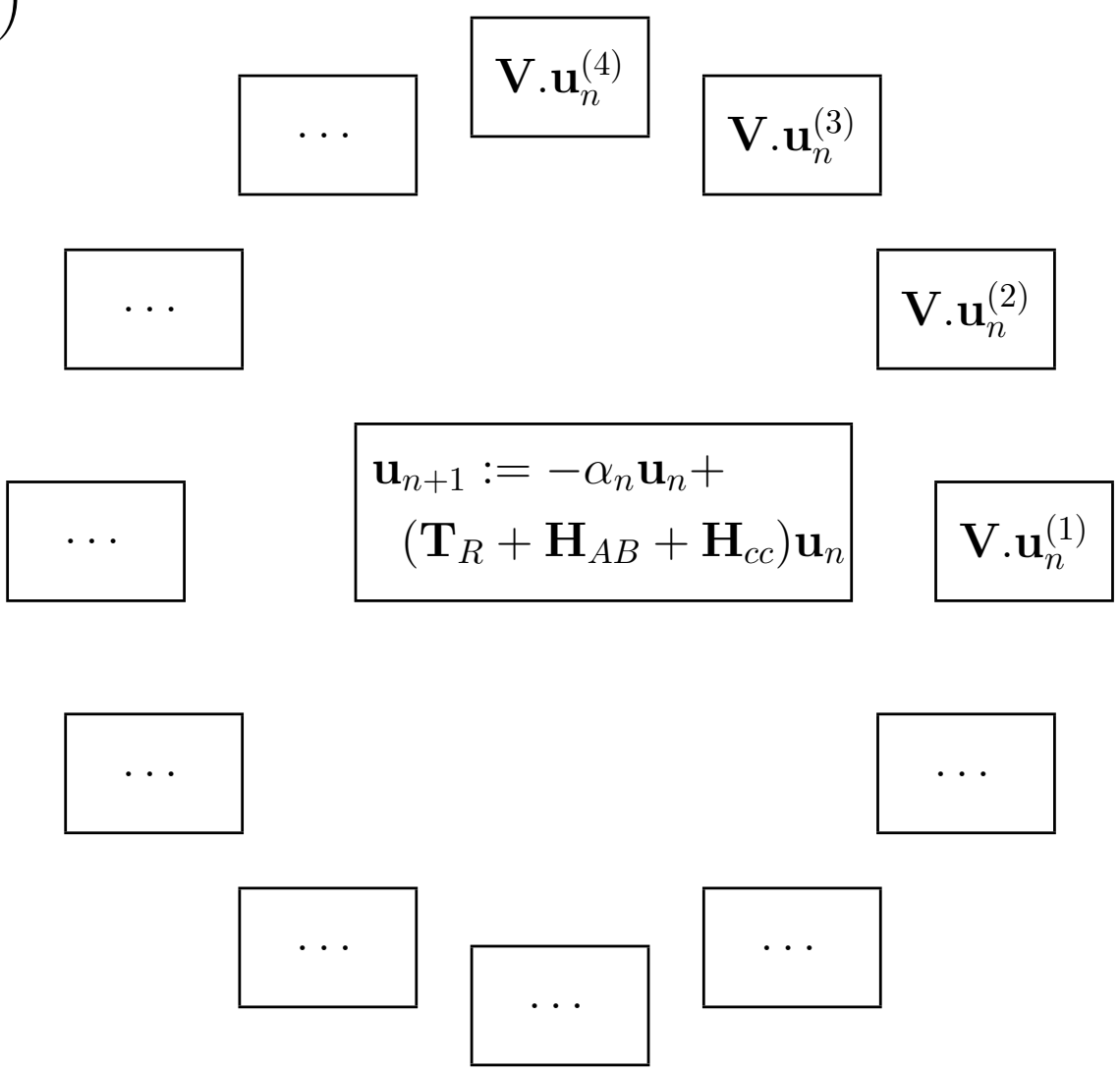


(c)

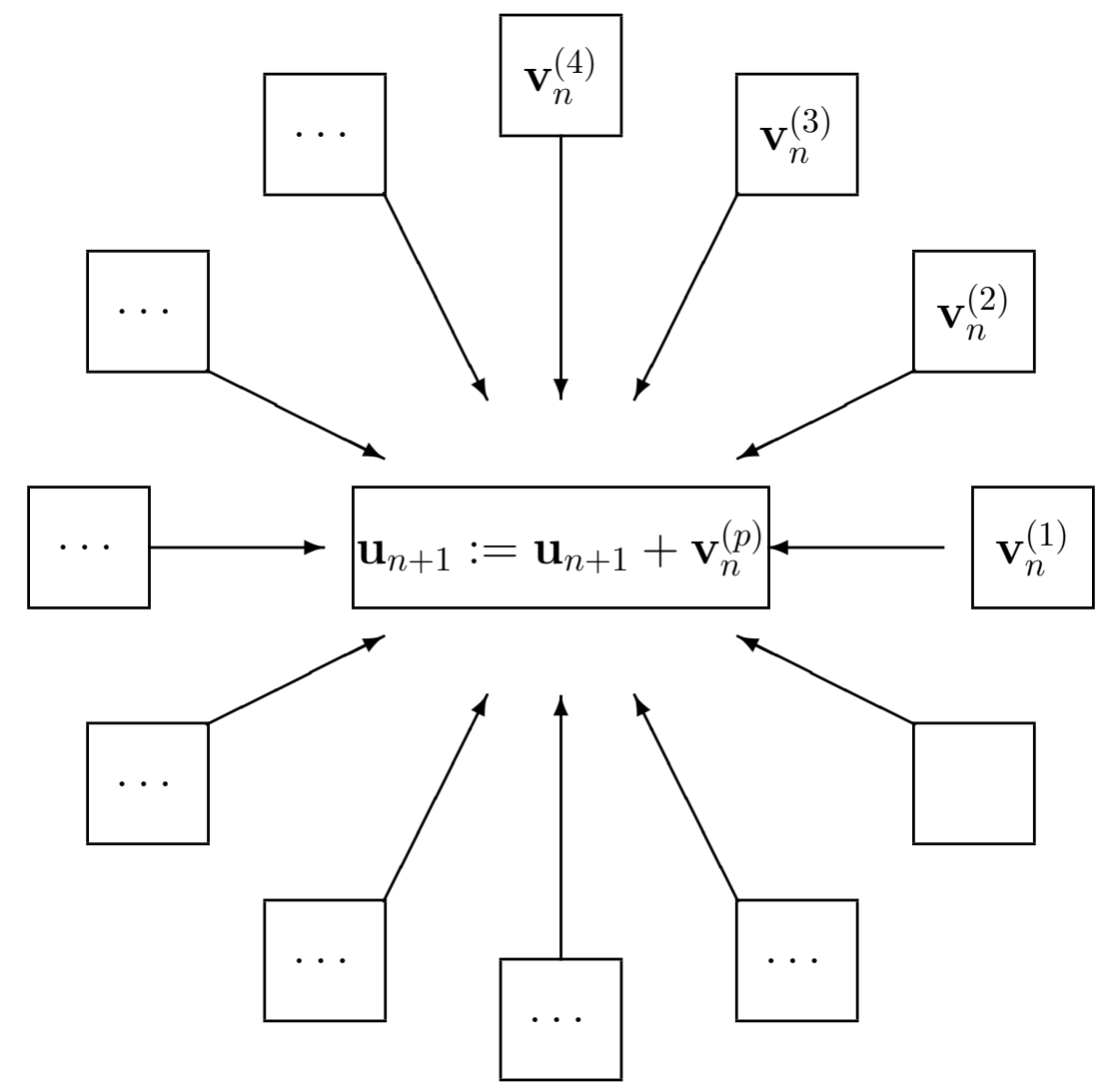

